\title{
Etude par simulation numérique d'un réacteur de synthèse de nanopoudres
}

\author{
Mohamed Amara - Mohammed El Ganaoui \\ SPCTS, UMR 6638 CNRS, Faculté des Sciences et Techniques \\ 123 Avenue Albert Thomas \\ F-87060 Limoges cedex \\ Mohamed.amara@unilim.fr
}

RÉSUMÉ. Dans le cas de la synthèse de poudres céramiques par pyrolyse laser, un modèle de calcul numérique a été développé. Ce modèle prend en considération les transferts d'énergie et les réactions chimiques, le but étant de comprendre les différents mécanismes responsables de la formation et de la collecte des poudres. Une étude du réacteur dans un régime turbulent a été réalisée dans le but de concevoir un réacteur industriel.

ABSTRACT. In the present work, a numerical model was developed for the simulation of the process of ceramic powder synthesis by the laser pyrolysis. It takes into account the flow, heat transfer and chemical reactions. The aim of this investigation is to develop a three dimensional model of the reactor and to characterize the influence of the various involved thermal parameters during the process. This will contribute to an improved fundamental understanding of the heat and mass transfer and fluid flow during the synthesis of ceramic nanopowdera. In addition, The reactor is studied under turbulent flow regime in order to provide first guidance of the design and the optimisation of the geometry of an industrial synthesis reactor.

MOTS-CLÉS: nanopoudres, nanocomposites, synthèse de nanopoudres par pyrolyse laser, mécanique des fluides numériques (MFN), thermique numérique, fluides réactifs, jets turbulents.

KEYWORDS: nanopowders, nanocomposites, laser induced formation of nanopowders, silicon, computational fluid dynamics (CFD), comupational heat transfer (CHT), reactive flow, turbulent jet.

DOI:10.3166/REMN.16.775-794 @ 2007, Lavoisier, Paris 


\section{Introduction}

L'évolution rapide du marché des céramiques de hautes performances stimule le développement de nouveaux procédés de synthèse susceptibles de produire des poudres ayant les propriétés suivantes : morphologie équiaxe, haute pureté chimique surtout au voisinage de la surface, taille moyenne de particules inférieure à $100 \mathrm{~nm}$, distribution étroite à un seul pic et une faible agglomération, pour abaisser la température et éviter un agent facilitant le frittage des matériaux (Ring, 1996). Les débouchés de ces poudres sont nombreux et variés: dans l'automobile, l'aéronautique, le spatial, le militaire (blindage, avion furtif...), la chimie (support de catalyseur, mousses inorganiques, pigments...) et l'électronique.

L'intérêt de ces poudres, devant servir de matière première pour la fabrication des matériaux, a trois explications majeures :

- plus une particule est petite, plus sa surface est grande par rapport à son volume. De ce fait, toutes les interactions entre la particule et son environnement sont exacerbées. C'est la raison pour laquelle les catalyseurs à base de nanopoudres sont plus efficaces ;

- quand on passe du micro aux nanopoudres, les grains pourraient être plus réguliers, voire équiaxes et avec une faible dispersion de taille. Ces poudres sont ainsi intéressantes pour l'élaboration de matériaux ;

- la réduction de la taille modifie fortement les propriétés des particules et des matériaux, ouvrant la voie à des composés plus performants ou innovants.

C'est sans doute la découverte de la ductilité des céramiques à base de $\mathrm{Si}_{3} \mathrm{~N}_{4}$ et la mise à profit de cette propriété pour l'élaboration de matériaux thermostructuraux par formage à chaud qui est la plus innovante (Wakai, 1991, Nieh et al., 1991). En effet, le phénomène de formage à chaud constitue un atout majeur, puisqu'il pourrait permettre de réduire le coût important de fabrication de certaines pièces de forme, lié à l'usinage délicat des matériaux durs à base de nitrure de silicium. La condition véritablement indispensable pour avoir un domaine ductile important pour les céramiques est une taille de grains inférieure au micromètre. La mobilité des grains au cours de la déformation est également facilitée par d'autres caractéristiques microstructurales, à savoir, la morphologie équiaxe des cristaux et la présence d'une phase liquide intergranulaire provenant des ajouts de frittage, qui sont indispensables à la densification des matériaux à base de nitrure de silicium.

Durant les vingt dernières années, un important travail a été effectué pour élaborer cette nouvelle classe de matériaux. C'est pourquoi, il y a eu un important développement des techniques de synthèse de poudres utilisant les différents états de la matière (gaz, solide, liquide). Ces recherches ont mis en avant des techniques axées sur les réactions en phase gazeuse. Parmi ces multiples procédés de synthèse de poudres par voie gazeuse, la pyrolyse laser s'avère un bon candidat pour l'obtention de poudres de taille nanométrique de haute pureté (Pratsinis, 1988). Le contrôle du procédé de synthèse nécessite une compréhension de plusieurs 
phénomènes physico-chimiques impliquant plusieurs disciplines: mécanique des fluides, thermodynamique, chimie, sciences pour l'ingénieur.

Dans le présent travail, l'étude porte sur la modélisation du procédé de synthèse des poudres SiCN par pyrolyse laser. Une étude par simulation numérique à l'aide d'un code industriel de CFD (Computational Fluid Dynamics) a été entreprise dans le but de mieux comprendre et d'aider au contrôle et à l'optimisation de la synthèse. En effet, une étude purement expérimentale sans interaction avec une approche par simulation s'avère délicate, du fait de la difficulté d'accès aux mesures à l'intérieur du réacteur pendant la synthèse (champ thermique, pression, vitesse...).

L'objectif ici est de développer un modèle 3D capable de décrire la dynamique des gaz réactifs pendant la synthèse et d'évaluer leur dépendance aux paramètres de contrôle (débit des gaz, intensité du laser, pression...) et aux caractéristiques de la réaction. Aussi, une étude du réacteur dans un régime laminaire est entreprise et étendue au régime turbulent dans le but d'exploiter la possibilité de l'utilisation de ce procédé à l'échelle industrielle.

\section{Mode de synthèse de nanopoudres par pyrolyse laser}

Parmi les procédés d'élaboration de poudres céramiques de type nanométrique, la synthèse par pyrolyse a été sélectionnée en raison de sa souplesse d'utilisation pour synthétiser des poudres multi-éléments à base de silicium (Musset, 1995, Doucey, 1999).

La méthode de synthèse de poudres nanométriques par pyrolyse laser a été développée par Haggerty (Cannon et al., 1982a, 1982b) dans les années quatre vingt au MIT (Massachusetts Institute of Technology). Elle résulte de l'interaction de flux croisés d'un laser $\mathrm{CO}_{2}$ dans le domaine infrarouge $(\lambda=10,6 \mu \mathrm{m})$ et de précurseurs gazeux ou liquides (via un aérosol) (figure 1). Une condition nécessaire est qu'il y ait résonance entre le spectre d'émission du rayonnement laser et le spectre d'absorption d'au moins un des réactifs. Cette interaction a pour conséquence une augmentation rapide de la température $\left(10^{6} \mathrm{~K} / \mathrm{s}\right)$ suivie d'un effet de trempe. En effet, après absorption des niveaux vibrationnels des molécules, l'énergie est redistribuée au système par collision, activant ainsi les réactions chimiques de décomposition. Le gaz atteint rapidement l'équilibre thermique. Les particules issues de cette méthode se caractérisent par une grande pureté, un contrôle de la stoechiométrie et une distribution en taille étroite et uniforme. En effet, du fait que la réaction soit très localisée, elle ne se trouve pas en contact avec les parois du réacteur. De plus, l'effet de trempe que subissent les particules, limite leur taille lors du processus de croissance, donnant ainsi des diamètres de quelques dizaines de nanomètres. En plus de la synthèse des nitrures et des carbures, cette technique permet la synthèse du $\mathrm{TiB}_{2}, \mathrm{~B}_{4} \mathrm{C}, \mathrm{ZrB}_{2}$, ainsi que des poudres métalliques (Weimer, 1997). 


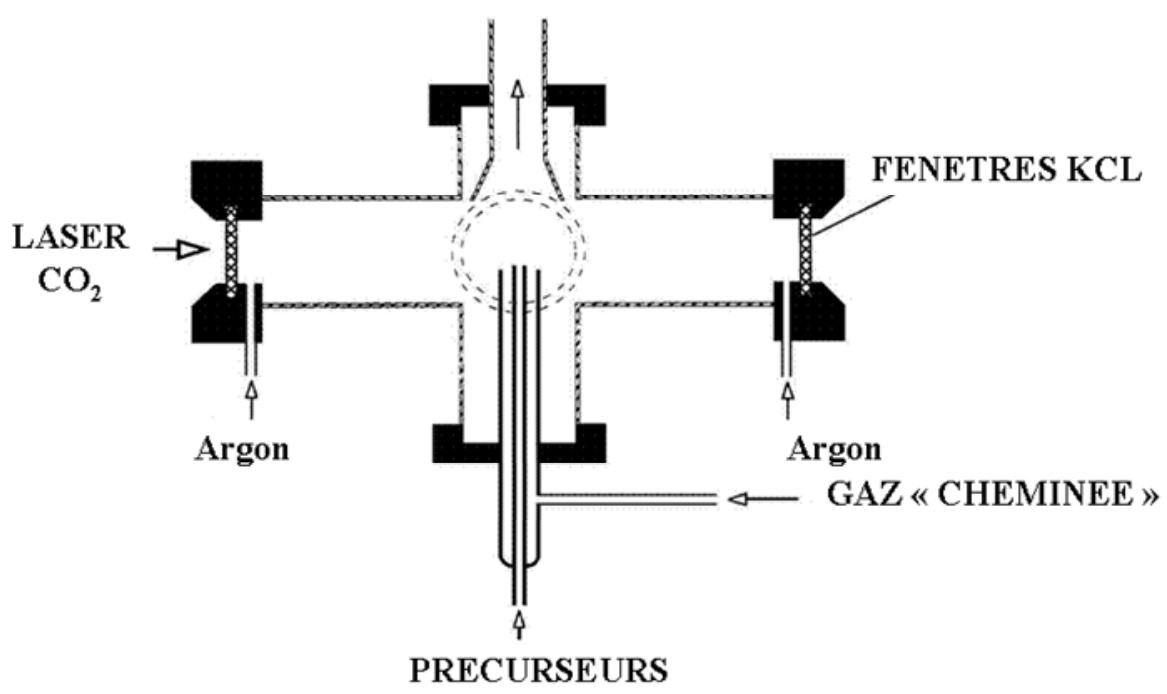

Figure 1. Représentation simplifiée de l'enceinte de réaction

L'étude des propriétés des matériaux issus des nanopoudres montre une sensibilité aux procédés de synthèse (Amara et al., 2004). Ceci inclut la nature des précurseurs utilisés (propriétés physico-chimiques), les paramètres de contrôle (débit des gaz, intensité du laser...) ainsi que les champs globaux (dynamique et thermique, pression...) et les paramètres locaux, au voisinage de la zone réactionnelle (température maximale, gradients locaux de température).

Par ailleurs, pour ce qui est de la production des poudres, il existe plusieurs modèles décrivant les différentes étapes responsables de la formation des particules (Ring, 1996). Le principe de base de ces modèles est que, après la décomposition rapide des précurseurs, il y a formation de particules de silicium, suivie par des processus de nitruration ou de carburation de ces derniers. Par conséquent, un modèle qui prendra en considération la formation du silicium peut être considéré suffisant pour décrire la dynamique de la synthèse par pyrolyse laser. Ainsi, ce travail se focalisera sur la synthèse du silicium.

Développer et contrôler un réacteur de synthèse par pyrolyse laser, implique la mise en place d'une méthodologie de quantification des effets des paramètres ajustables par l'expérimentateur. L'approche classique par plans d'expériences s'avère ardue du fait de la difficulté d'accès aux mesures. Pour cette raison, nous avons mené des simulations numériques au moyen d'un code industriel (ANSYSCFX) pour tenir compte en particulier de la source laser et des réactions chimiques ainsi que de la géométrie du réacteur. Cette étude s'est focalisée sur un réacteur de synthèse par voie gazeuse, ceci étant une première étape pour toute autre généralisation aux autres types de précurseurs. Egalement, pour une mise en place 
d'un réacteur à production industrielle, nous développerons une étude du réacteur dans un régime d'écoulement turbulent.

\section{Problème physique et mise en place des équations}

\subsection{Considération phénoménologique}

La plupart des précurseurs de poudres céramiques, possèdent une bande d'absorption dans le domaine infrarouge dans la gamme variant de $3 \mu \mathrm{m}$ à $30 \mu \mathrm{m}$. Ces fréquences de vibration coïncident avec la longueur d'onde d'émission des lasers infrarouges de puissance. Une condition nécessaire pour que le taux de réaction soit significatif est que l'énergie d'excitation soit de l'ordre de grandeur de l'énergie d'activation des réactions chimiques (typiquement de l'ordre de 100 à $380 \mathrm{kj} / \mathrm{mol}$ ). En réalité, plusieurs mécanismes physiques entrent en jeux pour induire l'absorption du rayonnement, ainsi que la décomposition des réactifs. Cependant, d'un point de vue macroscopique, l'absorption du rayonnement laser se fait suivant une loi de type Beer-Lambert (Haggerty, 1981);

$$
\mathrm{I}=\mathrm{I}_{0} \cdot \exp \left(-P \cdot \alpha_{a b s} . \Delta L\right)
$$

où :

$I_{0}$ : intensité initiale du faisceau laser [W]

$\alpha_{a b s}$ représente le coefficient d'absorption gaz/laser $[\mathrm{m} / \mathrm{N}]$

$P$ : la pression du gaz $[\mathrm{Pa}]$

$\Delta L$ : épaisseur optique du gaz $[\mathrm{m}]$

Il est à noter que le laser n'a pas de présence matérielle et donc pas d'effet sur l'écoulement des gaz. Néanmoins, il crée une dissymétrie lors de la décomposition des gaz. En effet, le gaz qui diffuse perpendiculairement au laser ne réagit pas, ce qui fait que la zone réactive ressemble plutôt à un ballon aplati dans la direction du laser. Ceci est très important pour l'élaboration du modèle, à savoir que le réacteur présentant un axe de révolution qui n'admet pas pour autant le développement d'un modèle à symétrie axisymétrique.

Comme déjà exposé précédemment, ce travail s'intéresse à la synthèse de poudres céramiques à base de silicium $\left(\mathrm{Si}_{2} \mathrm{Si}_{3} \mathrm{~N}_{4}, \mathrm{SiC}\right.$ et $\left.\mathrm{SiCN}\right)$. Celles-ci sont généralement synthétisées à partir du silane, avec d'autres gaz tels que l'ammoniac et/ou le méthane. L'utilisation du silane comme précurseur de silicium est liée d'une part, à la forte absorption de ce gaz au rayonnement IR à 10,6 $\mu \mathrm{m}$, du fait de ses liaisons $\mathrm{Si}-\mathrm{H}$. Celles-ci permettent d'augmenter la température de la flamme de pyrolyse et ainsi d'améliorer la décomposition des autres réactifs présents. D'autre part, ce gaz contient seulement du silicium et de l'hydrogène. 
Pan et al., (1987) ont montré que le coefficient d'absorption du silane dépend fortement de la pression (figure 2). Par ailleurs, on s'attend aussi à une influence de la température du fait du phénomène d'élargissement des raies d'absorption

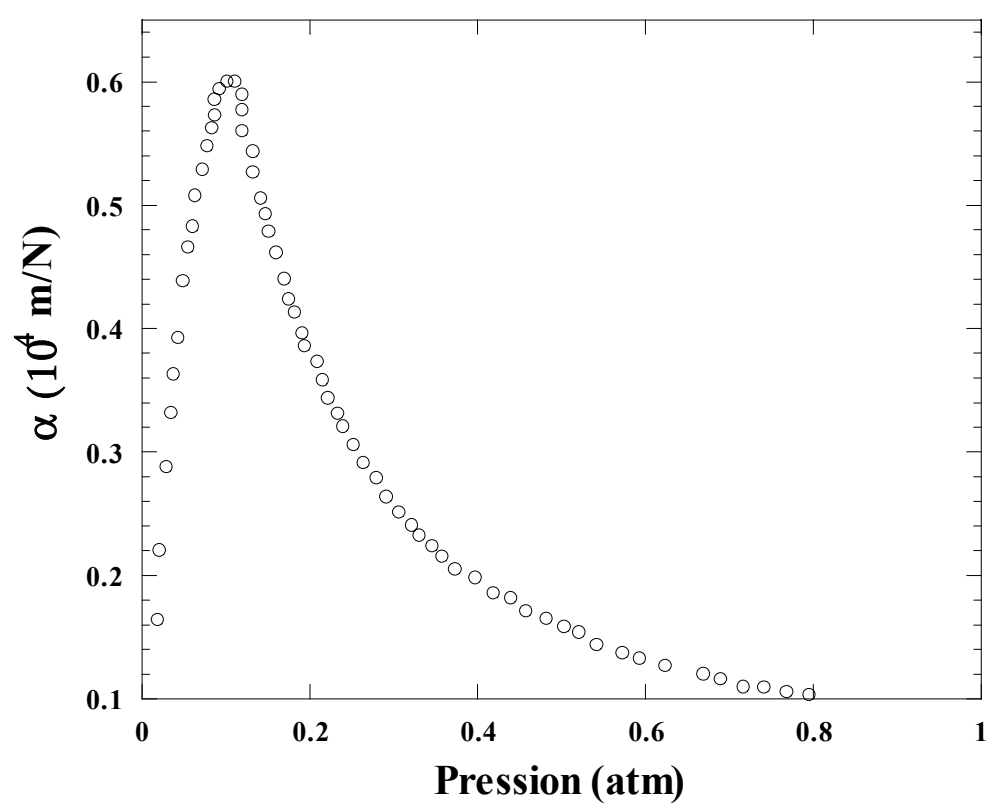

Figure 2. Variation du coefficient d'absorption du silane en fonction de la pression (Haggerty and Cannon 1981, Marra, 1983)

La réaction de décomposition thermique qui se produit vers les $700 \mathrm{~K}$ s'écrit :

$$
\mathrm{SiH}_{4}(\mathrm{~g}) \stackrel{700 \mathrm{~K}}{\longrightarrow} \mathrm{Si}(\mathrm{s})+2 \mathrm{H}_{2}(\mathrm{~g})
$$

Lors de la décomposition thermique du silane, la plupart des auteurs (Marra, 1983, Newman et al., 1979) ne révèlent pas la formation des combinaisons complexes correspondant aux hauts états du silane comme le $\mathrm{Si}_{3} \mathrm{H}_{8}$ ou $\mathrm{Si}_{3} \mathrm{H}_{6}$ etc. Ils suggèrent que la décomposition se produit uniquement par les réactions unimoléculaires, c'est-à-dire directement par la formation du silicium et de l'hydrogène. Coltrin et al., (1984) ont montré que cette réaction passe par 120 étapes, donnant naissance à plusieurs radicaux, tels que $\mathrm{SiH}_{2}$ ou $\mathrm{SiH}_{3}$. En outre, ils montrent qu'à partir de $900 \mathrm{~K}$, l'espèce majoritaire est le silylène qui contrôle ainsi la décomposition du silane suivant la réaction :

$$
\mathrm{SiH}_{4}(\mathrm{~g}) \rightarrow \mathrm{SiH}_{2}(\mathrm{~g})+\mathrm{H}_{2}
$$


avec un taux de réaction donné par la loi d'Arrhenius :

$$
\frac{\mathrm{d}\left[\mathrm{SiH}_{4}\right]}{\mathrm{dt}}=\left[\mathrm{SiH}_{4}\right] .5 \times 10^{12} \exp \left(\frac{-218 \mathrm{kj} / \mathrm{mol}}{\mathrm{RT}}\right)
$$

Il est à remarquer que ce que nous traitons ici concerne la décomposition thermique du silane et non pas sa décomposition par combustion. En effet, la combustion est réalisée à condition de mettre le carburant (silane) avec l'oxydant. Or dans le présent cas, il n'y a aucune condition de mélange avec l'oxygène, la principale condition de réaction est que l'énergie cédée au silane dépasse l'énergie d'activation $(\sim 220 \mathrm{kj} / \mathrm{mol})$.

\subsection{Formulation mathématique}

Le présent modèle tridimensionnel permet de résoudre les équations de conservation de la masse, de l'énergie et de la quantité de mouvement. Ces équations peuvent êtres écrites sous leur forme générale donnée par Patankar (1980) :

$$
\frac{\partial}{\partial t}(\rho \phi)+\nabla \cdot(\rho u \phi)=\nabla \cdot(\Gamma \nabla \phi)+S_{\phi}
$$

où $S_{\phi}$ représente le terme source de la variable $\phi$, et $\Gamma$ est le coefficient de diffusion correspondant. Dans le cas de l'équation d'énergie, le terme source est égal à celui donné par l'équation [1].

Pour traiter des réactions chimiques, nous supposons que les espèces se mélangent à l'état moléculaire. Par conséquent, on les représente par un champ scalaire de concentration (masse par unité de volume) des espèces. Ceci se traduit par une équation de transport donnée par :

$$
\frac{\partial C_{j}}{\partial t}+\frac{\partial}{\partial x_{i}}\left(C_{j} \cdot u_{i}\right)=\frac{\partial}{\partial x_{i}}\left(\Gamma_{j} \cdot \frac{\partial C_{j}}{\partial x_{i}}\right)+S_{j}
$$

$\mathrm{Cj}$ étant la concentration de l'espèce $\mathrm{j} ; \Gamma_{j}$ est la diffusivité massique moléculaire et enfin, $\mathrm{Sj}$ est le terme source, significatif de la création/destruction de l'espèce $\mathrm{j}$ : celui-ci est donné par l'équation [4]. Toutefois, il existe un couplage entre le terme source de l'équation de l'énergie et des espèces chimiques. En effet, seul le silane absorbe le rayonnement laser, et de ce fait, le terme de pression apparaissant dans l'équation [1] représente la pression partielle de silane.

La description de l'écoulement en régime turbulent pour le présent réacteur se heurte au problème de l'absence de mesures expérimentales relatives à l'écoulement en régime turbulent. La plupart des études analytiques et numériques ne se focalisent 
sur l'étude du jet que dans sa région auto similaire (Bailly and Comte- Bellot, 2003). Par ailleurs, dans le cas des jets libres, la valeur critique du nombre de Reynolds de transition n'est pas bien définie, et peut varier entre 1000 et 1500 (Mhiri et al., 1998). Néanmoins, le jet est entièrement turbulent quand $\operatorname{Re}>10^{5}$ (Roef, 2000).

Nous avons utilisé pour décrire le phénomène de turbulence, un modèle standard $k-\varepsilon$. Ce modèle est basé sur le concept de l'isotropie de la turbulence. Il correspond à la résolution de deux équations de transport ([7] et [8]), l'une pour l'énergie cinétique de la turbulence $k$, et l'autre pour la dissipation de cette énergie $\varepsilon$ (Wolfgang et al., 2002). En effet, Roef (2000) a montré que l'utilisation d'un modèle $k-\varepsilon$ standard (utilisant les constantes données par le tableau 1) est suffisante pour l'investigation de jets circulaires libres et des flammes de diffusion rencontrées dans les laboratoires.

L'hypothèse de base du modèle $k-\varepsilon$, est que les effets de la viscosité moléculaire sont négligeables. Il permet une description de la turbulence à haut nombre de Reynolds. L'inconvénient de ce modèle est l'introduction de constantes empiriques (tableau 1).

$$
\begin{aligned}
& \frac{\partial(\rho k)}{\partial t}+\frac{\partial\left(\rho u_{j} k\right)}{\partial x_{j}}=P_{k}-\rho \varepsilon+\frac{\partial}{\partial x_{j}}\left(\Gamma_{k} \frac{\partial k}{\partial x_{j}}\right) \\
& \frac{\partial(\rho \varepsilon)}{\partial t}+\frac{\partial\left(\rho u_{j} \varepsilon\right)}{\partial x_{j}}=C_{\varepsilon 1} \frac{\varepsilon}{k} P_{k}-C_{\varepsilon 2} \rho \frac{\varepsilon^{2}}{k}+\frac{\partial}{\partial x_{j}}\left(\Gamma_{\varepsilon} \frac{\partial \varepsilon}{\partial x_{j}}\right)
\end{aligned}
$$

Où :

$$
\begin{aligned}
& \Gamma_{k}=\mu+\frac{\mu_{t}}{\sigma_{k}} \\
& \Gamma_{\varepsilon}=\mu+\frac{\mu_{t}}{\sigma_{\varepsilon}}
\end{aligned}
$$

Dans ces équations, $P_{k}$ représente la génération de l'énergie cinétique turbulente due aux gradients moyens de vitesse :

$$
P_{k}=\mu_{t}\left(\frac{\partial u_{i}}{\partial x_{j}}+\frac{\partial u_{j}}{\partial x_{i}}\right) \frac{\partial u_{i}}{\partial x_{j}}+\frac{2}{3} \rho k \delta_{i j} \frac{\partial u_{i}}{\partial x_{j}}
$$


La viscosité turbulente est donnée par :

$$
\mu_{t}=C_{\mu} \rho \frac{k^{2}}{\varepsilon}
$$

Les constantes sont données dans le tableau suivant (Wolfgang et al., 2002) :

\begin{tabular}{|ccccc|}
\hline$\sigma_{k}$ & $\sigma_{\varepsilon}$ & $\mathbf{C}_{\varepsilon 1}$ & $\mathbf{C}_{\boldsymbol{\mu}}$ & $\mathbf{C}_{\varepsilon 2}$ \\
\hline $\mathbf{1 , 0}$ & 1,3 & 1,44 & 0,09 & 1,92 \\
\hline
\end{tabular}

Tableau 1. Constante du modèle standard $k-\varepsilon$ (Documentation du code CFX 5)

\subsection{Méthode de résolution}

Pour notre étude, notre choix s'est porté sur le logiciel industriel ANSYS-CFX. Les équations de conservation décrites ci-avant sont discrétisées en utilisant une méthode éléments finis basée sur l'approche volumes de contrôles (Schneider et Raw, 1987). Ainsi, ANSYS-CFX maintient une grande partie de la flexibilité géométrique des méthodes éléments finis aussi bien que les propriétés importantes de conservation de la méthode volume finie. La maille peut se composer de tétraèdres, de prismes, pyramides, et éléments hexaèdres. Par ailleurs, le maillage a été raffiné autour des zones de forts gradients. Ceci permet de bien "reconstruire » la flamme de réaction.

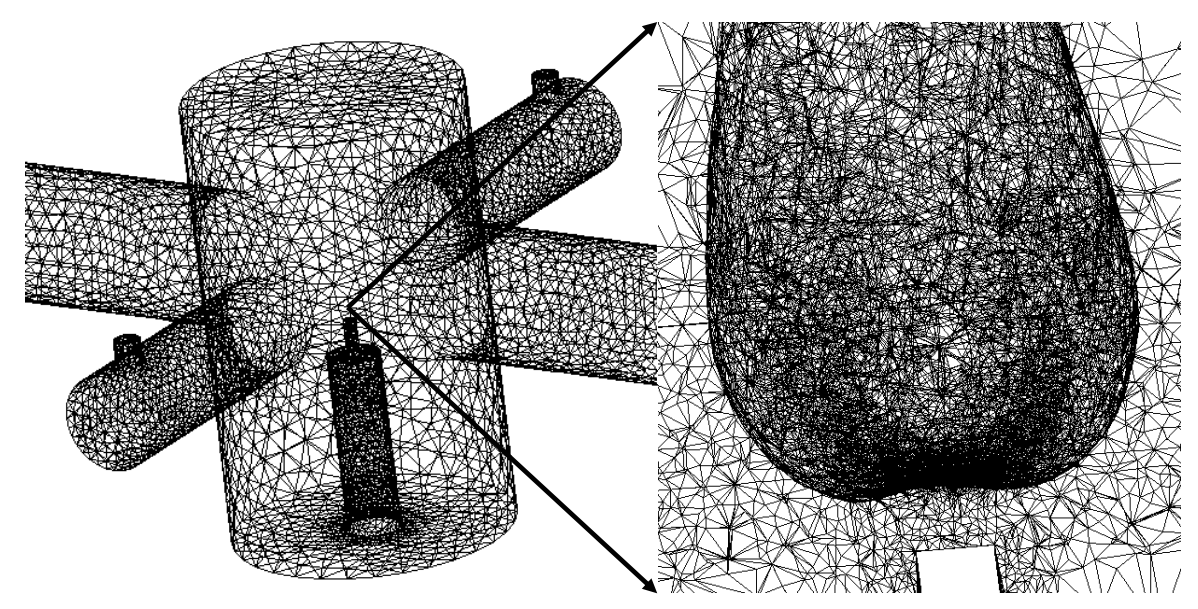

Figure 3. Maillage du réacteur et finement autour de la flamme. La surface, indique l'iso-surface à température égale à $700 \mathrm{~K}$ 
ANSYS-CFX utilise un solveur de type AMG (Raw, 1994) (méthode multigrille algébrique).

Le logiciel permet également de choisir plusieurs façons de prendre les conditions aux limites selon qu'il s'agisse de condition de Dirichlet, Neuman ou Robin. Dans la présente configuration, nous avons utilisé trois types de conditions aux limites :

- conditions d'entrée : dans le présent cas, il est plus judicieux d'utiliser une condition de type de "Mass flow boundary», cette condition est souvent choisie lorsque le débit est connu. L'utilisateur doit alors spécifier un débit en $[\mathrm{kg} / \mathrm{s}]$ pour qu'il soit imposé à l'écoulement sous forme d'une condition de Neumann sur la vitesse,

- conditions de sortie : en ce qui concerne la condition de la sortie du fluide, il est plus physique d'utiliser une condition sur la pression (à moins de connaitre les débits ou les vitesses de sortie du fluide), celle-ci étant fixée par une condition de Dirichlet. En effet, expérimentalement, la sortie du réacteur est connectée à une pompe qui permet de maintenir une pression constante,

- conditions de paroi : pour les parois, nous avons utilisé une condition de nonglissement (vitesse tangentielle nulle).

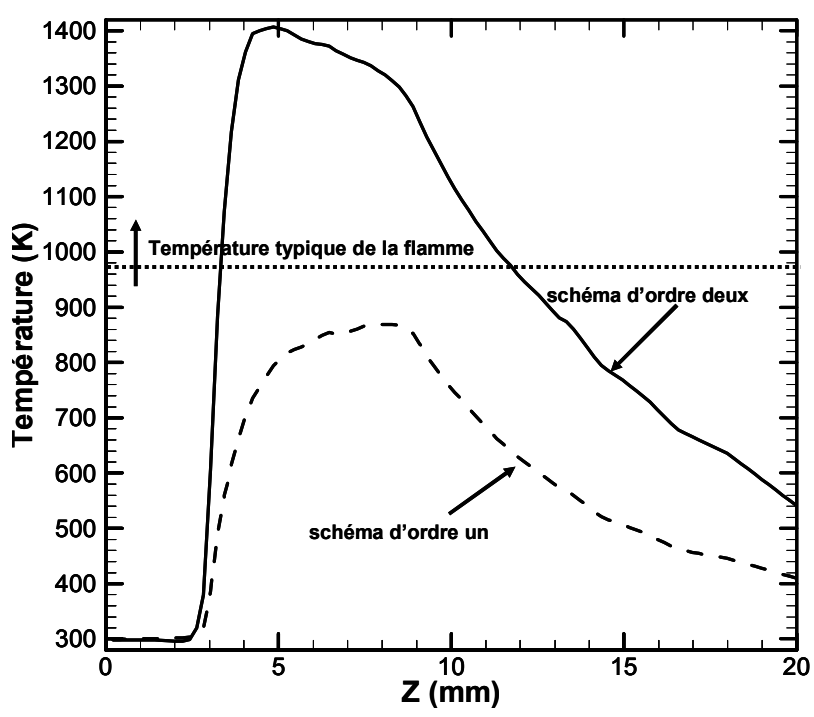

Figure 4. Comparaison entre les températures obtenues avec les différents schémas de résolution

Plusieurs schémas de discrétisation spatiale sont disponibles sous CFX. Des schémas de second, premier ordre ou hybrides. Leur utilisation dépend des opérateurs composant les équations différentielles du système. Ainsi pour les termes de convection, nous avons utilisé un schéma du second ordre. En effet, les schémas du premier ordre ont tendance à sous-estimer les transferts de chaleur, qui sont d'autant 
plus importants que la convection domine. La figure 4 montre la température le long de l'axe du jet obtenue avec deux schémas de résolution. Le schéma d'ordre deux permet une meilleure prédiction de la température. Enfin, pour ce qui est des termes de diffusion et des termes sources, ils sont discrétisés à l'aide d'un schéma centré du second ordre, ceci en considérant les deux mailles amont et aval.

\subsection{Validation}

Avant d'aborder les résultats des simulations, une étape de validation des paramètres numériques est présentée. Le nombre de nœuds a été varié jusqu'à ce que la solution ne dépende plus du maillage. Ainsi, deux grandeurs physiques ont été sélectionnées, à savoir la température et la vitesse (figure 5).
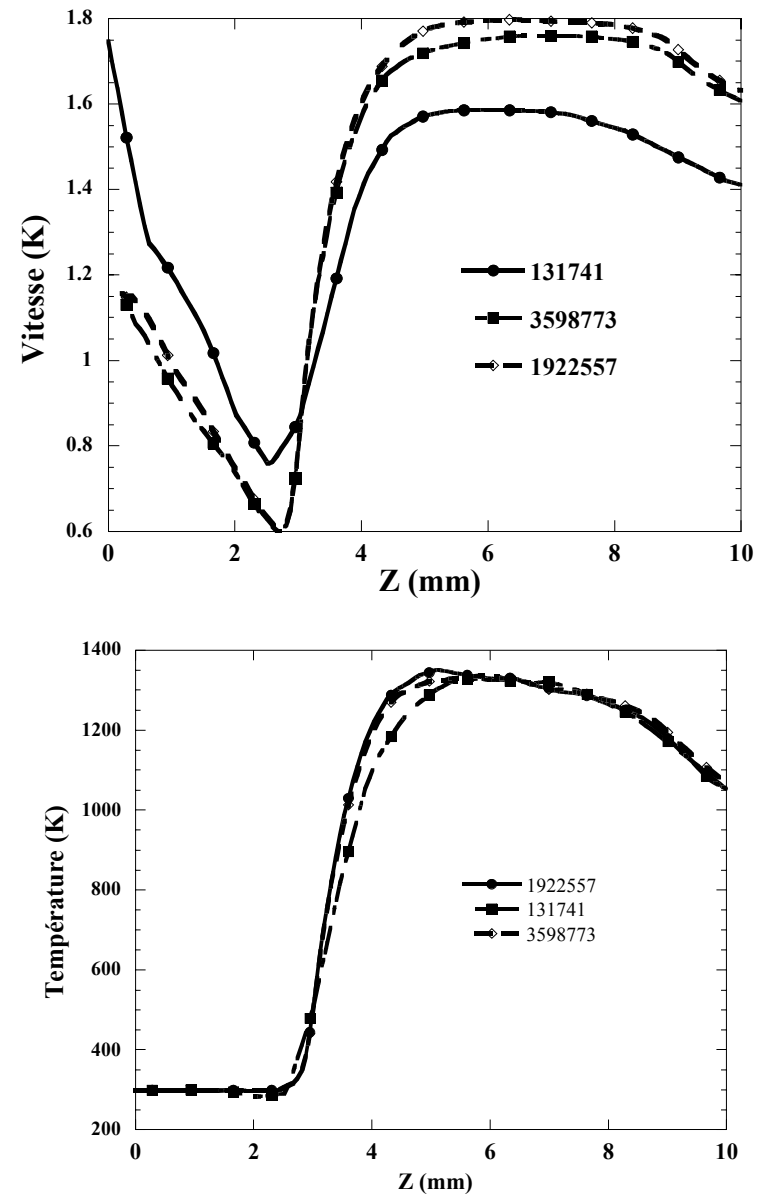

Figure 5. Solutions obtenues pour la température et vitesse pour plusieurs nombres de nœuds du maillage 
On remarque que la vitesse est plus sensible au maillage au voisinage de la buse d'injection des gaz. Pour la suite des calculs, nous utiliserons un maillage avec un nombre de nœuds de l'ordre de $2 \times 10^{7}$ permettant un bon rapport entre CPU et la précision du calcul.

\section{Résultats et discussion}

Pour les présentes simulations, les débits des gaz (réactifs et vecteur) ont été considérés comme conditions aux limites. La pression de l'enceinte est maintenue constante pendant la synthèse. Les paramètres utilisés sont donnés dans le tableau 2.
Pression totale de l'enceinte ( $\mathrm{atm}$ )
$0,2-1,0$
Densité volumique de puissance laser $\left(\mathrm{W} / \mathrm{m}^{3}\right)$
$1,910^{9}$
Débit du silane $\left(\mathrm{cm}^{3} / \mathrm{min}\right)$
$30-2000$

Tableau 2. Paramètres expérimentaux du modèle

\subsection{Etude globale de l'écoulement}

Lors d'études précédentes (Amara et al., 2006), nous avons montré le rôle prédominant de la pression pour les phénomènes de transferts d'énergie et de masse : la pression agit d'une part, sur la température maximale et d'autre part, sur le gradient thermique. Ces résultats sont en bon accord avec les résultats expérimentaux donnés par (Marra, 1983). Par ailleurs, le mélange des réactifs est plus efficace à basse pression. Cette première étude (réacteur à l'échelle du laboratoire) nous a permis d'avoir une étude globale des phénomènes de transports dans le réacteur de synthèse. Pour une installation industrielle, la présence d'un mode opératoire en écoulement turbulent n'est pas à écarter. Dans le domaine industriel, la plupart des réacteurs se trouvent dans un régime d'écoulement fortement turbulent. Pour le présent réacteur, la source de turbulence est générée par l'impulsion initiale du jet des gaz réactifs.

La figure 6 donne le profil de l'énergie cinétique turbulente le long de l'axe du jet pour différentes valeurs du débit du silane. Ces courbes présentent un comportement similaire en fonction de $z / D$ ( $D$ étant le diamètre de la buse d'injection $\mathrm{du}$ gaz réactif) comparées à celles données par les mesures expérimentales de jets turbulents (Roef, 2000). Il est intéressant de noter la présence d'un maximum d'énergie turbulente à une position indépendante du débit. Par ailleurs, il a été également observé que la présence du laser n'affecte pas la variation des grandeurs turbulentes le long du jet, seule leur intensité est modifiée. Ainsi, 
(Habli et al., 2001) ont montré que dans le cas des jets turbulents, le régime établi est atteint à une distance de la buse de $z / D=50$, ceci, quel que soit le profil initial de vitesse. Par ailleurs, la formation des poudres se situe dans une zone comprise entre $\mathrm{z} / \mathrm{D}=1$ et 5 . En conséquence, les processus de croissances pour le réacteur considéré se situent dans un régime d'écoulement laminaire.

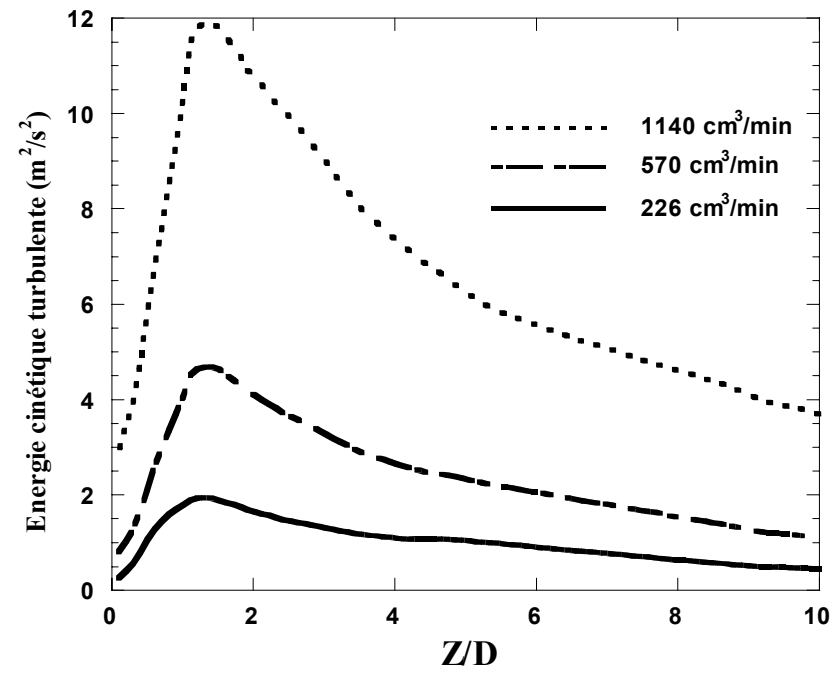

Figure 6. Profil de l'énergie cinétique turbulente $k$, le long de l'axe du jet pour différents débits de silane

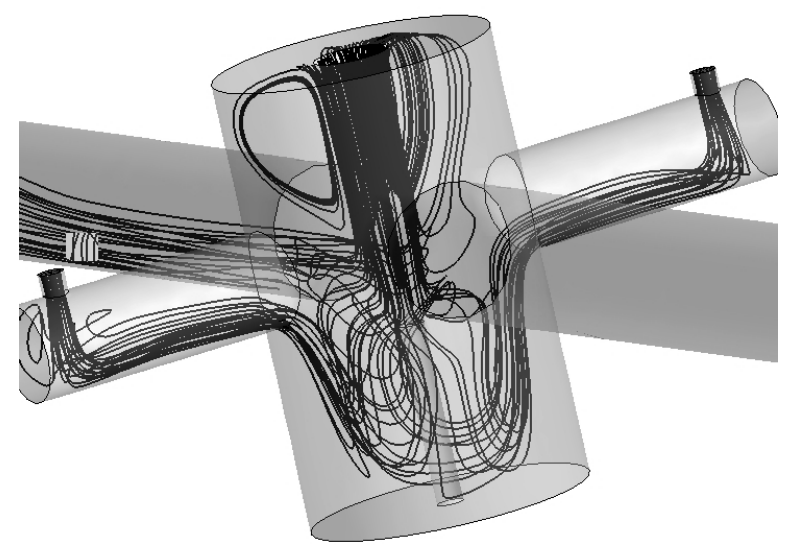

Figure 7. Ligne de courant dans le réacteur $3 D$ et vue de haut. $P=0,2$ atm; $\mathrm{SiH} 4=38 \mathrm{~cm}^{3} / \mathrm{min} ; \mathrm{Ar}$ (gaz vecteur) $=1000 \mathrm{~cm}^{3} / \mathrm{min} ; \mathrm{Ar}$ (fenêtres) $=400 \mathrm{~cm}^{3} / \mathrm{min}$ 
Travailler à haut débit de gaz réactif, pose un autre problème technique qui concerne la collecte des poudres. En effet si on observe la figure 7, on remarque un écoulement complètement perturbé avec présence de vortex au voisinage de la sortie des poudres (vers le collecteur). Nous avons noté que ce type d'écoulement est dû aux gaz secondaires (fenêtre de visualisation et conduit du faisceau laser).

A l'origine, ces gaz sont injectés pour protéger les hublots (fenêtre en $\mathrm{KCl}$ ) d'éventuelles poudres chaudes (les particules sont thermalisées à la température de flamme $\sim 1500 \mathrm{~K}$ ), qui peuvent par choc thermique briser ces verres. Les simulations montrent que l'annulation de ces gaz entraîne à haut débit une dissymétrie du champ thermique, voir la brisure de la flamme. En effet, la figure 8-a-b-c, montre le champ thermique pour plusieurs débits de silane ( $\mathrm{P}=0,2 \mathrm{~atm})$ en absence d'injection de gaz aux fenêtres. On remarque l'effet du 'souffle' du jet sur la flamme à partir de $1140 \mathrm{~cm}^{3} / \mathrm{min}$ de silane, on note la présence d'un effet d'asymétrie du laser. Car seul le gaz qui diffuse dans le sens du laser continue à interagir (figure 8d).

Les simulations numériques montrent que ce phénomène disparait à haute pression. Ceci indique que c'est plutôt l'interaction des jets fenêtres avec le gaz vecteur qui est responsable de l'écoulement dans le réacteur.
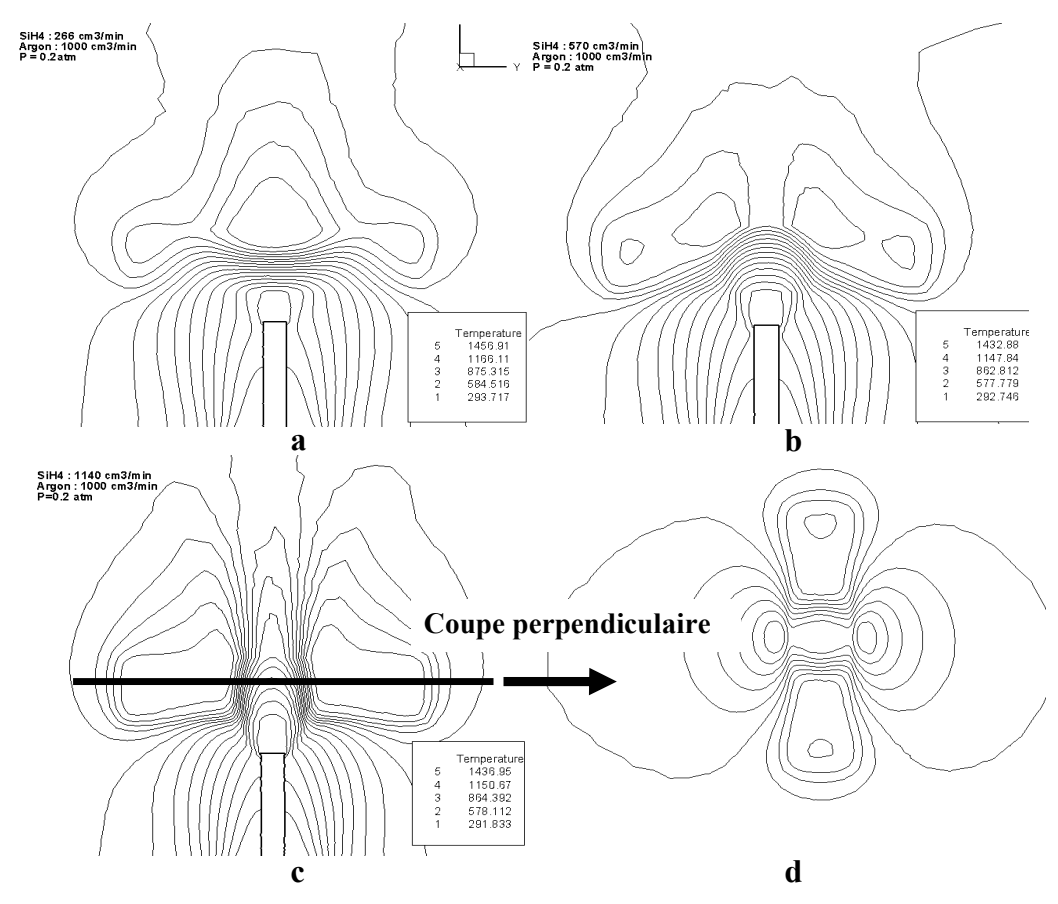

Figure 8. Champs de température dans le plan du jet $(a, b, c)$ et perpendiculaire au plan du jet à $z=7 \mathrm{~mm}($ d). Le débit de l'argon des quatre fenêtres est nul 
Pour pallier ce problème, nous avons augmenté la taille de la buse du gaz gaz vecteur (donc diminué sa vitesse à débit constant). Ainsi on conserve la quantité d'argon injectée dans le réacteur mais avec une vitesse de gaz confluent plus basse. Nous avons augmenté également le diamètre de sortie du réacteur pour drainer le maximum de poudres. En effet, les résultats de simulations numériques ont montré que doubler le diamètre de la sortie du réacteur permettait d'atténuer la formation de vortex en sortie. La figure 9 montre les lignes de courant dans le réacteur pour un débit de silane trente fois plus grand que celui donné par la figure 7. Nous remarquons la disparition des structures tourbillonnaires à la sortie. Le calcul montre que ces structures persistent à haute température quelle que soit la géométrie de la buse. En effet, les forces de flottabilité et la différence de densité entre les gaz sont plus importantes à haute pression (le gaz vecteur se trouve dans la zone plus chaude que les gaz fenêtre).

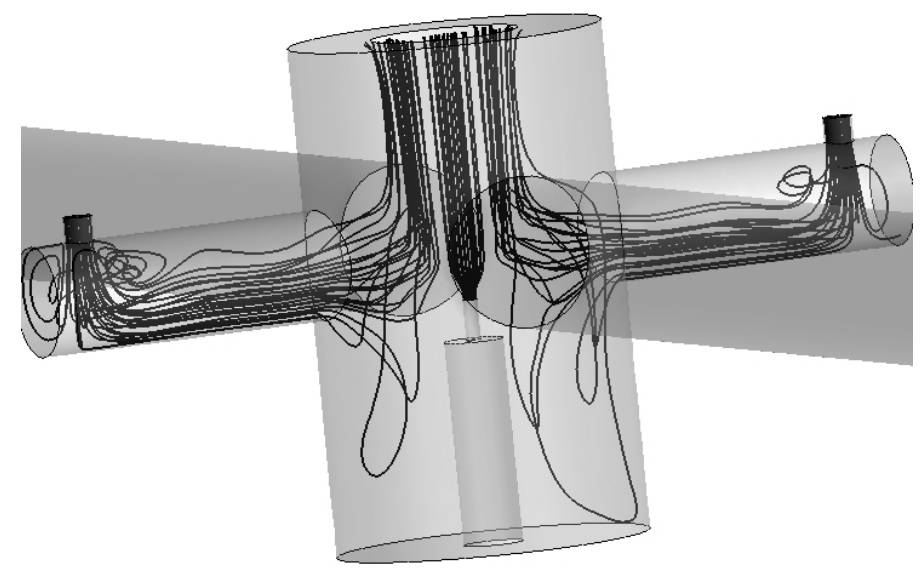

Figure 9. Ligne de courant pour un réacteur avec une buse argon de $16 \mathrm{~mm}$. Conditions opératoire: $P=0,2 \mathrm{~atm}, \mathrm{SiH}_{4}=1140 \mathrm{~cm}^{3} / \mathrm{min} ; \mathrm{Ar}$ (gaz vecteur) $=1000 \mathrm{~cm}^{3} / \mathrm{min}, \mathrm{Ar}$ (fenêtres) $=2000 \mathrm{~cm}^{3} / \mathrm{min}$

Il apparaît donc que la dynamique des gaz à l'intérieur du réacteur est bien régie par l'interaction des débits des gaz fenêtres et du gaz vecteur, et que cette interaction est d'autant plus importante à haute pression. Ces observations sont en bon accord avec nos résultats précédents (Amara et al., 2006) où nous avons montré que le mélange des gaz est plus efficace à basse pression dû à la diminution des forces de frottement.

\subsection{Stratégie pour la synthèse}

Le fonctionnement durable du procédé d'une manière fiable nécessite une stabilité de la flamme et des écoulements favorables à la formation et à la collecte. 
Par ailleurs, pour obtenir des quantités importantes de poudres, il va falloir augmenter considérablement la quantité de gaz réactif. L'augmentation du débit (à buse d'injection fixée à $2 \mathrm{~mm}$ ) est illustrée sur la figure 10. La température le long de l'axe du jet ainsi que la fraction massique du silane sont reportées pour deux valeurs du débit de gaz : $266 \mathrm{~cm}^{3} / \mathrm{min}$ et $570 \mathrm{~cm}^{3} / \mathrm{min}$.

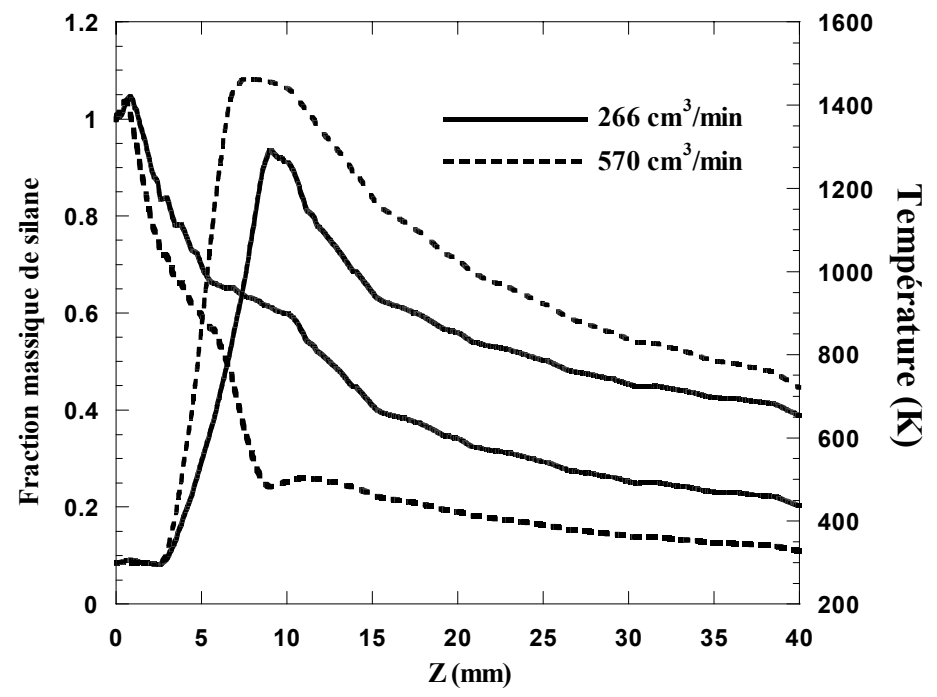

Figure 10. Température et fraction massique de silane le long de l'axe du jet pour deux débits de gaz réactif

Nous remarquons une diminution de la température le long de l'axe du jet avec le débit. Ceci peut être expliqué par deux phénomènes couplés: une vitesse plus grande a pour effet de diminuer le temps de séjour des réactifs à l'intérieur du laser. Ainsi, la quantité de silane qui va réagir sera elle aussi diminuée (figure 10). Ces deux phénomènes combinés impliquent une diminution du gradient thermique à l'entrée de la zone laser $(\mathrm{z}=3 \mathrm{~mm}$ sur la figure 10$)$, ce qui n'est pas sans conséquences sur les caractéristiques des poudres, telles que leur surface spécifique ou leur cristallinité. Une solution consiste à augmenter la taille de la buse d'injection des réactifs, nous avons donc doublé son diamètre. La figure 11 montre le nouveau champ de température dans la zone réactive, ainsi que l'évolution de la température le long de l'axe du jet. Nous remarquons une augmentation à la fois de la température et de la taille de la flamme, ce qui est attendu, vu que la quantité de réactifs a été augmentée. 
Une coupe de la flamme dans le plan perpendiculaire à l'axe du jet (figure 11 en haut à droite) au niveau du laser, montre que l'étendue de cette zone est légèrement plus grande que le diamètre du laser (cylindre noir au centre de la figure 11), ce qui pose problème au niveau de l'irradiation du gaz. En effet, augmenter le diamètre de la buse des gaz réactifs a pour effet d'élargir le jet. Dans ce cas, une partie du gaz ne sera pas irradiée par le faisceau laser. Ceci indique, qu'il faut utiliser un faisceau de plus grand diamètre $(>6 \mathrm{~mm})$, grâce à un jeu de lentilles de focalisation. L'évolution de la température le long de l'axe du jet (figure 11) montre un autre effet indésirable à savoir qu'à la sortie du réacteur (ici $\mathrm{z}=70 \mathrm{~mm}$ ), le gaz n'est pas refroidi à la température ambiante. Ceci pose le problème du fonctionnement de ce dernier, car, initialement ce procédé a été conçu pour travailler à parois froides. En augmentant le débit des gaz vecteurs (fenêtres plus la buse du gaz réactif) jusqu'à un débit de $3000 \mathrm{~cm}^{3} / \mathrm{min}$, ceci a permis de thermaliser les parois du réacteur (à température ambiante), sans créer de perturbations au niveau de l'écoulement. Aussi, il est nécessaire de redimensionner le présent réacteur pour une application industrielle.

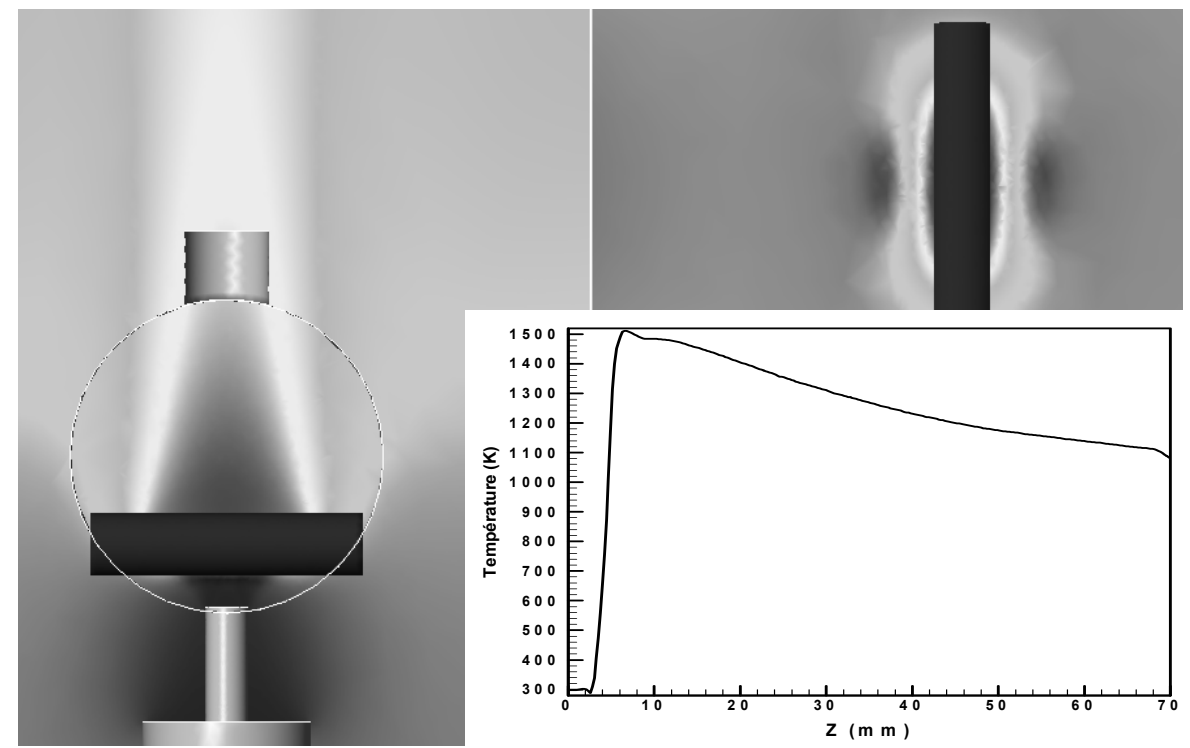

Figure 11. Champs thermiques près de la buse des réactifs. $\mathrm{SiH}_{4}: 1140 \mathrm{~cm} 3 / \mathrm{min}$, Ar (gaz vecteur) : $1000 \mathrm{~cm}^{3} / \mathrm{min}$, Ar (fenêtres) : $2000 \mathrm{~cm}^{3} / \mathrm{min}, P=0,2 \mathrm{~atm}$

Par ailleurs, les travaux de Kusters et al., (1995) ont montré que la présence de la turbulence est une condition indispensable pour la fabrication de poudres de bonne qualité. Or, nous avons montré dans les résultats précédents que cette turbulence est créée au-delà de la zone de formation des poudres. Par conséquent, il faut envisager 
un moyen pour créer artificiellement cette turbulence. Une des méthodes possible peut consister à utiliser un jet circulaire.

\section{Conclusion}

Développer et contrôler un réacteur de synthèse par pyrolyse laser, implique la mise en place d'une méthodologie de quantification des effets des paramètres ajustables par l'expérimentateur. C'est pourquoi nous avons mené des simulations numériques suite à l'intégration de développements spécifiques au problème à un code industriel (ANSYS-CFX) pour prendre en compte en particulier la source laser et les réactions chimiques ainsi que la géométrie du réacteur. Cette étude s'est focalisée sur un réacteur de synthèse par voie gazeuse, ceci étant une première étape pour toute autre généralisation aux autres types de précurseurs.

Les précédents résultats nous ont permis de mettre en évidence l'importance des phénomènes de transfert de chaleur et de masse dans l'ensemble du réacteur sur les caractéristiques de la zone réactive. Dans le présent travail, nous avons étendu le modèle au cas du réacteur fonctionnant dans un régime turbulent à haut débit, ceci étant indispensable pour toute utilisation du procédé à l'échelle industrielle. Toutefois, aux présents débits, nous avons dû redimensionner l'enceinte pour limiter les effets de circulation et éviter la présence de certains vortex, qui peuvent d'une part, perturber la formation des poudres, et d'autre part, diminuer la quantité acheminée vers le collecteur.

Par ailleurs, cette étude nous a permis d'avoir une idée globale sur l'écoulement du réacteur et les effets réels de l'interaction mutuelle des différents gaz (fenêtres d'observation et le gaz vecteur), ceci n'étant pas accessible expérimentalement. Aussi, pour augmenter le rendement du procédé, nous avons dû redimensionner les buses d'injection des gaz.

Enfin, pour ce qui est de la formation des poudres de silicium, le fait que la température calculée n'excède pas la température de fusion du silicium, confirme un mode de formation par collision et non pas par coagulation de gouttelettes. Par ailleurs, le régime de l'écoulement étant laminaire dans la zone réactive, il favorise la formation de poudres de petite taille du fait d'un temps de séjour réduit. Aussi, la formation des poudres multi-éléments $(\mathrm{Si} / \mathrm{C} / \mathrm{N})$ passe par deux étapes (formation $d u$ silicium puis sa nitruration ou sa carburation). Par conséquent, une stratégie pour obtenir des poudres de bonne qualité (homogène, distribution en taille réduite) consiste à séparer les deux étapes de formation. Par exemple, en séparant les voies d'injection du gaz silane avec les autres précurseurs de carbone et/ou d'azote. Ainsi, on favorisera la formation de fines particules de silicium, et dans ce cas, la taille finale des poudres est limitée par les différents temps caractéristiques de diffusion entre éléments $\mathrm{Si}-\mathrm{C}$ et $\mathrm{Si}-\mathrm{N}$. 
Dans le cas de nouvelle conception d'un réacteur, il est souhaitable d'utiliser un jet circulaire pour augmenter l'efficacité de la réaction et l'homogénéité des poudres.

Ces résultats laissent envisager un possible fonctionnement du procédé de synthèse en régime turbulent à condition de contrôler la transition, le temps des bouffées turbulentes et le temps de séjour des poudres dans la zone réactionnelle.

\section{Bibliographie}

Amara M., El Ganaoui M., "Simulation of Heat and Mass Transfer inside a Nanopwders Synthesis Reactor", Progress in Computational Fluid Dynamics, vol. 6, n ${ }^{\circ}$ 6. p. 371-278.

Amara M., Dez R., Foucaud-Raynaud.S., Bahloul-Hourlier D., Goursat P., Herlin-Boime N., Besson J.-L., "Fabrication and creep Behavior of $\operatorname{SiCN}(\mathrm{O})$ Nanocomposites", Silicates Industriels, Special Issue, 89, 2004, p. 281-285.

Amara M., El Ganaoui M., Hourlier D., "A preliminary study on the nanopowders synthesis: the role of the flow inside the reactor", Journal Mécanique \& Industrie, 7, 2006, p. 131-137.

Bailly C., Comte-Bellot G., Turbulence, CNRS édition, 2003.

Cannon W. R., Danforth S., Haggert J. S., Marra R. A., "Sinterible Ceramic Powders From Laser-Driven Reactions: II, Powder Characteristics and Process Variables", Journal of the American Ceramics Society, 65, 1982a, p. 330.

Cannon W. R., Danforth S. C., Flint J. H., Haggert J. S., Marra R. A., "Sinterible Ceramic Powders From Laser-Driven Reactions: I, Process Description and modelling", Journal of the American Ceramics Society, 65, 1982b, p. 324.

colocated variables - 1. Computational procedure, Numerical Heat Transfer, 11, p. 363-390.

Documentation du code CFX 5, User Manuel.

Doucey B., Nanocomposites $\mathrm{Si} 3 \mathrm{~N} 4 / \mathrm{SiC}$ : Stabilité thermique et densification de poudres $\mathrm{SiCN}$ (Al, O) synthèse par pyrolyse laser; Comportement au fluage, Thèse de Doctorat, Université de Limoges, 1999.

Habli S., Mhiri H., El Golli S., Le Palec G., Bournot P., «Etude numérique des conditions d'émission sur un écoulement de type jet axisymétrique turbulent », Int. J. Therm. Sci, 40, 2001, p. 497-511.

Haggerty J. S., Cannon W. R., Pleneum-Press, 1981, p. 165-241.

Kuster K. A., Pratsinis S. E., Strategies for control of ceramic powder synthesis by gastoparticle conversion, Powder Technology, 85, 1995, p. 79-91.

Marra R. A., Homogenous nucleation and growth of silicon powder from laser heated gaz phase reactants, PhD MIT, Cambridge, 1983.

Mhiri H., El Golli S., Le Palec G., Bournot P., «Influence des conditions d'émission sur un écoulement de type jet plan laminar isotherme ou chauffé », Rev. Gén. Therm, 37, 1998, p. 898-910. 
Michel E., Coltrin Robert J., Miller James A., "A Mathematical Model of The Coupled Fluid Mechanics and Chemical Kinetics in a Chemical Vapor Deposition Reactor", $J$. Electrochem Sos., 131, 1984, p. 425-34.

Musset E., Synthèse de poudres nanométriques à base de silicium, carbonate et azote par couplage aerosol-laser et leurs caractérisation, Thèse de Doctorat Université Paris XI, 1995.

Newman C. G., O’Neal H. E., Ring M. A., Leska F. and N S., "Kinetics and mechanism of the silane decomposition", Int. J. Chem. Kinet, 11, 1979, p. 1167.

Nieh T. G., Wadswoth J., Wakai F., "Recent advances in Superplastic Ceramics and Ceramic Composites", International Materials Reviews, 33, 1991, p. 146-161.

Pan E. T-S., Flint J. H., Liang J. M., Adler. D. and Haggerty J. S., "Model for gaz-laser interaction: application to thermally activated laser-induced chemical vapor deposition", Applied Optics, 26, 1987, p. 70-75.

Patankar Suhas V., "Numerical Heat Transfer and Fluid Flow", Hemisphere Publishing Coprporation, 1980.

Pratsinis S. E., "Vapor Synthesis of Ceramic Powders", Ceramic Transactions, 12, 1988, p. 227-239.

Raw M., "Robustness of coupled algebraic multigrid for the Navier-Stokes equations", AIAA, 1994, 96-0297.

Ring T. A., "Fundamentals of ceramic powder processing and synthesis", Academic Press, 1996, p. 255-306.

Roef L., The numerical simulation of turbulent jets and diffusion flames, Eindhoven University Press, The Netherlands, 2000.

Schneider G. E., Raw M. J., Control volume finite-element method for heat transfer and fluid flow using collocated variables. II: Application and validation, Numerical heat transfer, 11, 1987, p. 391-400.

Wakai F., "Superplasticity of Ceramics", Ceramics International, 17, 1991, p. 153-163.

Weimer A. W., Carbide, Nitride and Boride, Materials synthesis and processing, CHPMAN \& HALL, 1997.

Wolfgang V., Esch. T. and Menter F., ANSYS CFX, 2002. 